\title{
Interferences between psychology and psychiatry - vulnerability versus mental fragility in schizophrenia and delusional disorder
}

\author{
Andrei Coșman ${ }^{\mathrm{a}}$, Alexandru Pană ${ }^{\mathrm{a}}$, Ioana Rădulescu*b \\ ${ }^{a}$ Faculty of Psychology and Educational Sciences, 90 Panduri Str., 050663, Bucharest, Romania \\ b"Alex. Obregia" Cljnical Hospital for Psychiatry, 10 Berceni Str., 041914 Bucharest, Romania
}

\begin{abstract}
The aim of this paper is a didactic one, namely the separation of concepts of vulnerability in psychosis, with cognitive, thinking, affective, relational and motivational patterns of patients with schizophrenia, explaining the diagnosis of emotional schizophrenia after DSM VI, which although has a long history, is still a controversial one and excluded from DSM V. The paper also discusses brief psychoses. Vulnerability in psychoses is both psychological, hereditary, genetic or potentially related to the pathology of the neurotransmitters involved, and it is also based on concepts such as: social rejection, disabilities (predominantly auditory), immigration, inability to adapt to cultural patterns, an emotional climate characterized by expressing excessive emotions, an onset with significant stressors or a postpartum hormonal situation.
\end{abstract}

Keywords: vulnerability; mental fragility; psychosis; schizophrenia; delusional disorder; prognosis; schizoaffective disorder; brief psychotic disorders; precipitating factors

\section{Introduction}

Mental health problems are not the result of personal weakness, lack of character or lack of education. Mental illness affects young and old, men and women, and does not take into account nationality or ethnic roots, level of education or financial status (Tereanu, 2011).

The object is dual, psychiatric and psychological. Vulnerability is a concept closer to the broad area of schizophrenia, while that of mental fragility is closer to the psychological concept of stress. It is currently known that in mental illness the judgment is based on the stress - diathesis model, and this one has particular connotations depending on the premorbid personality and/or the onset or aggravating circumstances of delirium.

The hypothesis is directly related to the stress-diathesis model and the load of each, on the specific case in question, having also the extreme possibility of schizophrenia described in Predescu, namely that category D which represents a percentage of $20 \%$ of patients who recover with a considerable defect, but also the functioning of the delusional disorder or the spectacular return of the Brief Psychotic Disorders.

\section{Vulnerability in psychoses}

The etiopathogenesis of psychoses (both in terms of schizophrenia and in terms of delusional disorder) operates with models of vulnerability. There is an integrative concept: explaining the variability of experimental and clinical data. The biopsychosocial approach is also known, according to which the occurrence of

\footnotetext{
* Corresponding author.

E-mail address: ioana.radulescu95@gmail.com
} 
schizophrenia is determined by the complex interaction between different factors. We are talking, therefore, about multifactorial models, which open new perspectives, proposing explanatory hypotheses both for the etiopathogenesis of diseases and in terms of clinical and evolutionary variability.

The concept of vulnerability takes into account the complex interactions between individual susceptibility factors and environmental risk factors. These interactions are at the origin of an increased risk and possibly of clinical manifestations (Trifu, 2020; Neagu, 2012).

\subsection{Zubin and Spring's model. Vulnerability to stress}

Endogenous and / or exogenous factors can produce a state of crisis in any individual. Depending on the intensity of stressors and the threshold of individual tolerance, the crisis is contained by homeostasis or causes a psychotic episode. Vulnerability is described in a trait / state relationship with the episode, hence the interest in differentiating between status markers (episode-specific) and trait markers (vulnerability-specific). The model allows the identification of vulnerability through potential markers.

Thus, there are three main categories of markers:

1) vulnerability markers - identified before the first episode; independent of clinical evolution; have family origin or not. They are opposed by protective markers (the presence of which in the healthy relatives of patients plays a protective role against the occurrence of a psychotic episode). The onset of a psychotic episode arises from the interaction between the degree of individual vulnerability, stressors and moderating or protective variables.

2) status markers - which appear during the episode.

3) residual markers - which are identified during the episode, persist during remission and may be the result of disease or extrinsic factors, such as iatrogenic.

We can distinguish two major components of vulnerability:

- innate (genetic) vulnerability, which is reflected in the body's neurophysiology;

- $\quad$ acquired (external) vulnerability, which is loaded by the influence of traumas, perinatal complications, family experiences, the complexity of relationships in adolescence, other life events.

Genetic diathesis can be triggered by certain stressors. Strategies that allow an individual to cope with difficulties will be tested. The individual ability to adapt (coping) + the support offered by the socio-family network are moderating factors as opposed to the stressful ones. The failure of these mechanisms causes disturbances of psychological hoemostasis, which can no longer be controlled by the individual. The subject's tolerance or vulnerability threshold results from the balance between the effectiveness of the moderating agents and the impact of stress.

The health (or disease) in an individual is dependent on the following factors: physical, sociocultural, educational, economic, specific to the "ecological niche" it occupies. The model articulates the different ethiopathogenic hypotheses, clinical data and highlights the interactions between biological and environmental factors, privileging the dynamic and evolutionary aspect of the disorder, an aspect neglected by the criteriological approaches.

Cognitive impairment - attention brings into question the most promising candidate markers for explanation. Psychotic patients (and especially those at high risk for schizophrenia) have poor performance in neurocognitive tests, especially those that assess components of prosexic processes.

Only a few of them meet sufficient criteria of vulnerability indicators: the Continuous Performance Test, which appears modified regardless of the stage of the disease, other cognitive disorders of psychotic patients, considered as probable indicators of vulnerability (low performance in tests sensitive to alteration of frontal functions, especially the Wisconsin Card Sorting Test, which supports the hypothesis of an involvement of the frontal cortex in vulnerability to schizophrenia). Functional imaging studies performed during the application of cognitive tests support the 
existence of a link between vulnerability to schizophrenia and disorders in the prefrontal regions.

\subsection{Electrophysiological disorders}

They bring together several types of potential markers: disorders of eye tracking movements, changes in endogenous brain evoked potentials, abnormalities of the electrodermal response.

\subsection{Conclusions}

Vulnerability includes traits that are expressed through perceptible, sometimes measurable changes that exist before the onset of psychosis symptoms. Only some of the subjects at risk have traits of vulnerability. Vulnerability is considered a necessary but not sufficient condition for the development of psychosis, which will occur in a minority of vulnerable people.

\section{Schizophrenia. General aspects and description}

For a good understanding of what in psychiatry does not mean schizophrenia, but other related psychotic disorders, we consider it necessary to recall the psychodynamic characteristics of schizophrenia. Schizophrenia is an intensely studied disorder in the history of psychiatry. Before using diagnostic manuals such as DSM, different psychiatrists used different criteria to describe schizophrenia in its complexity (Trifu, 2020; Sîrbu,2020).

Kraepelin's criteria are: attention disorders, hallucinations, thinking disorders, language disorders, affective disorders, behavioural disorders, obedience, echolalia, echopraxia, acting-outs, catatonia.

Bleuler's criteria are divided into two categories: fundamental disorders: thinking disorders, affection disorders, ambivalence, autism, disorders of subjective feelings of the Self, disorders of volition and behavior; accessory symptoms: hallucinations, delusional ideas, memory disorders, personality changes, language disorders, somatic symptoms, catatonic symptoms, affective symptoms.

Schneider's criteria, based on which the symptoms of schizophrenia can be classified into two ranks: first-order symptoms: sounding of thought, arguing voices, commentary voices, feelings of somatic passivity, delusional perceptions, "manufactured" actions, feelings; second degree symptoms: delusion intuition, other perceptual disorders, perplexity, mood swings, emotional impoverishment.

Crown's criteria, who describes three types of schizophrenia:

Type I: positive symptoms, good premorbid personality, acute onset, normal computed tomography examination, good response to treatment, good evolution over time.

Type II: negative symptoms, deficient premorbid personality structure, insidious onset, computed tomography examination that reveals abnormalities, resistance to treatment, poor evolution over time (Rujescu, 2004).

Type III: disorganized speech and behaviour, cognitive and attention defects, both positive and negative symptoms, many manifestations that simulate negative symptoms, but are the consequence of medication.

\subsection{Perception disorders}

People with schizophrenia have various perceptual disorders. In addition to the simple classification of delusional ideas and hallucinations, we can also talk about: unusual perceptual sensations, commentary voices, audible thoughts (pseudohallucinations), experiences of somatic passivity, withdrawal of thoughts, influence of thoughts, thoughts recording, delusional perceptions (absence of contact with the world through the natural senses), "manufactured" actions, feelings. 
Hallucinations can take many forms, such as auditory, visual, olfactory, gustatory, somatic / tactile (psychosensory or pseudohallucinations) and kinaesthetic (sensations of altered body pattern).

\subsection{Formal thinking disorders}

These fall into the spectrum of negative symptoms. Among them, in schizophrenia, we have: affective flattening, inadequate affection, alogia, avolition, anhedonia, social inattention.

In the field of positive symptoms, language, thinking and communication disorders: derailment, tangentiality, inconsistency, lack of logic, circumstantiality, speech pressure, distracting speech, clanging (mechanical associations by assonance, rhyme, contiguity), neologisms and word approximations, perseverance, echolalia, decreased ability to use abstract concepts - the interpretation of proverbs.

People with schizophrenia have very different behavioural and affective patterns from normal ones, and this disorder affects many aspects of the patient's life. Those in question may have patterns of withdrawal, irritability, or aversion, they often have problems with personal care, friends, performance, and their feelings and perceptions appear dissociated from each other. They may feel bodily sensations as unfamiliar, they may feel perplexity, lack of concentration and alienation. To cope with these destabilizing experiences, people with schizophrenia respond through innate patterns of adaptation, such as denial, symbolic explanation, or irrational explanations. (Waller, 1990) These people were probably shy, introverted, with a poor relationship and showed cognitive precocity in some areas and unexplained deficiencies in others. People with schizophrenia may also have an increased vulnerability to stress and may experience frequent state of fears (Weickert, 2004).

The attitudes of patients with schizophrenia are also affected. Some of them may be bizarrely intrusive, others may be hostile and oppose, may be perplexed, confused, naive, or "empty" (Saha, 2005). They often forget to thank, smile, or answer questions. The qualities that make a person unitary are poorly integrated, those in question not having a clear idea where their body, mind and influence end. They may feel that they are fused with external objects or that they are completely disintegrated. Therefore, we can speak of a loss of the boundaries of the Self, which is manifested by the loss of belonging to the Self, the inability to differentiate the Self - nonSelf, the doubling of the Self, depersonalization - derealization, delirium of xenopathic influence and gender ambiguity (Williams, 2007; Lefter, 2020). People with schizophrenia are also emotionally affected. The condition of these patients may be flat, blunt, apish- ridiculous or labile - inappropriate. They may be inconsistent with the content of speech and may provide emotionally inappropriate responses. Cadence, modulation of communication is disrupted, a phenomenon also known as aprosodia. People with schizophrenia may also have a series of bizarre emotions, such as: exaltation, the impression that they are omnipotent, the feeling of loneliness in the universe, ecstatic religious states, a terrifying apprehension of being physically disintegrated or anxiety about the imminent destruction of the universe.

\section{General information on Brief Psychotic Disorders}

Brief psychotic disorder is a disorder that occurs as a result of an obvious stressful event in a patient's life. This disorder can be triggered even if the patient does not have significant risk factors.

The disorder may be characterized by one or more of the following symptoms: delirium, hallucinations, disorganized speech. This diagnosis should not be applied when the patient has cultural / mystical beliefs acquired in the environment from which it comes that can be confused with the aforementioned symptoms.

From clinical point of view: delusional ideas with brutal occurrence (under 48 hours), delusional polymorphic ideas and disorganization, along with hallucinations and illusions; mental disorganization: emotional (anxiety, anger) and affective lability (euphoria, depression), sudden changes in psychomotor behaviour (agitation, 
prostration), feelings of depersonalization, derealization, favourable evolution. No psychiatric history other than other acute psychotic episodes

The diagnosis of brief psychotic disorder should be avoided when a diagnosis of schizophrenia, schizoaffective disorder, mood disorder with psychotic elements can better explain the symptoms experienced. General medical conditions, sometimes affected by a somatic illness (Grigoriu, 2018; Popa, 2019) and potential substance use must also be taken into account. Compared to other schizophrenia disorders, the brief psychotic disorder lasts at least a day and a maximum of one month. After this episode, the patient has a complete recovery and often finds it difficult to explain his thoughts and behaviours during the psychotic episode.

The disorder has three forms: with significant stressors (brief reactive psychosis), without significant stressors, with postpartum onset (in the next four weeks).

\subsection{Evolution and prognosis}

Magnan spoke of "the delusional hot flush is without consequences".

Favourable prognostic factors are: sudden onset, short duration, precipitating psychotraumatic factors, clinical features such as affective symptoms, confusion, prebmorbid: good functioning, absence of schizoid features, hereditary antecedents: relatives more with affective disorders than with schizophrenia disorders

Unfavourable prognostic factors are: insidious onset, absence of a precipitating, clinical factor: delusional polymorphism and of the thymic disorders are not very obvious; symptoms of schizophrenia predominate, less anxiety, persistence of delirium, duration of the episode (with or without treatment) of more than four weeks, premorbid: schizoid features, poor functioning, family medical history schizophrenia spectrum disorders

The symptoms of this disorder resolve very quickly. For a more effective recovery, the patient can follow a psychotherapeutic approach, to understand the significance of the events and to develop coping mechanisms.

Vulnerability is given by: personality disorders: paranoid, schizotypal, histrionic, borderline, narcissistic; socio-cultural and family factors, cross-cultural psychiatry, psychotraumatic factors. The event must be viewed through the impact on the subject, the crisis is important because it signals the fragility of the person

The average age of manifestation is $20-30$ years.

Evolution:10-50\% single episode, $30-50 \%$ multiple similar episodes, $10-30 \%$ evolve to schizophrenia, 5-20\% evolve to mood disorders (bipolar disorder).

Therapeutic principles: Mandatory hospitalization (car and hetero-aggressive risk). Elimination of an ingestion of substances (hallucinogens, amphetamines, alcohol, cocaine) or of a complicated withdrawal (alcohol, barbiturates). Elimination of an organic confusional syndrome. Correction of a possible hydro-electrolytic imbalance. Pharmaceutical treatment.

\subsection{Therapeutic strategies}

Treatment of the acute episode: Minimum duration 4-6 weeks. Dose adjustment according to symptoms and side effects; possibly changing the antipsychotic.

Continuation of treatment: In case of remission after 4-6 weeks continue 6-12-24 months with possible dose reduction. In case of therapeutic failure: compliance with the treatment is checked, possibly the dose is increased, the antipsychotic is changed. After the third episode, treatment will continue for several years (5 years). In case of cyclicity, timoregulators are recommended. (Stahl, 2013)

\subsection{Psychotherapy}

The association of a psychotherapy to the antipsychotic treatment gives better results. This is not done during the acute episode. Many episodes are precipitated by psychotraumatic events. More often young patients, faced with such an experience, need support, including family therapy (Pulver, 1992). 
Clinical example 1: A 15-year-old girl developed a brief psychotic disorder after learning that her mother was suffering from breast cancer and after her mother called her from a railway junction, threatening to throw herself in front of the train. In order to solve the mother's suicide attempt, it was necessary for the police and a team of negotiators to intervene. This event was extremely stressful for the patient who did not have a loaded diathesis for mental illness or other previous psychotraumatic agents, but strong enough to cause a psychotic intensity disorder.

Example 2: 24-year-old who developed a brief psychotic disorder following unfortunate life events. During that time, his grandfather had a stroke with severe complications, which led to his hospitalization and death. The patient was called to a party the night his grandfather died, where he consumed psychoactive substances (possibly as a defence mechanism). The patient's symptoms developed around these events, having a long-term (of almost 3 years) prodromal phase, which culminated in a complete psychotic episode with delusional ideas and hallucinations.

Other psychotic disorders:

Psychotic disorder not otherwise specified (PNOS)

This disorder can rather be described as a category for those psychotic disorders that do not meet the diagnostic criteria for other psychotic disorders. These disorders include:

Autoscopic psychosis: A rare disorder, characterized by hallucinations through which the patient "sees his/her body at a distance", versions of his body, often distorted in a caricatural manner.

Cotard Syndrome: It is also called "walking corpse syndrome". The patient has delusional ideas of denial, can be considered immortal and cursed to suffer for eternity.

Capgras Syndrome: It is a disorder characterized by delusional ideas according to which significant people in the patient's life have been replaced by impersonators who want to hurt him/her. This disorder can be thought and investigated from a neurological perspective.

\section{Schizophreniform disorder}

\subsection{History}

It is discussed by Langelfeldt 1939, as a heterogeneous group, with symptoms similar to those of schizophrenia, but with good evolution. Traits: good premorbid adaptation, sudden onset, frequently the presence of a precipitating factor, favourable prognosis. After DSM-IV-R, symptoms of schizophrenia are characteristic, but the duration is between one month and six months, after which total remission occurs.

\subsection{Diagnostic criteria}

For a significant period from one month the following symptoms are present: delusional ideas, hallucinations, disorganized speech, catatonic behaviour and negative symptoms; it is important to note that only one of these symptoms is sufficient, if imperative or commentary voices are present, discussing the patient's thoughts or actions, or if the delusional ideas are bizarre.

In terms of structuring, we are talking about an insidious prodromal stage, to which the active phase and later, the residual phase are added - usually the active phase taking place over a period of about a month, preceded by that prodromal stage, which can also last up to one month and being followed by the residual period which can last up to four months. All three of these components translate into a complete episode, which, depending on how it varies, lasts more than a month, but less than six months.

Unlike schizophrenia, in schizophreniform disorder, the residual period means a gradual transition to normality, on the background of a drug treatment (antipsychotic), but a very important factor in this transition is psychotherapy, with emphasis on strengthening the patient's personality, more precisely by metabolization and integration of that psychotic episode. 
It is important to note that this diagnosis can be used for patients whose personal characteristics have a good outlook for the future (for example the age advantage) and who are in the first episode of this kind. This also helps to destigmatize the patient, denoting optimism in relation to the possibility of occurrence of future episodes - in which case the diagnosis of schizophrenia will be chosen. Otherwise, the emphasis on the fact that the disorder is remitted will represent an important element for raising the patient's morale and the possible avoidance of disorders that could be truncated by the consequences of stigma.

Some clues regarding an optimistic prognosis are: the rapid development of the episode, as well as a relatively short prodromal stage; an evolution of this episode simultaneously with the presence of states of confusion or perplexity (spatial, temporal, general); decreased ability to resonate emotionally, which is very characteristic of schizophrenia (emotional blunting);

This diagnosis involves the exclusion of Schizoaffective Disorders and Mood Disorders.

Another condition for this diagnosis is the absence during the active period of a major depressive episode, manic or mixed. Or, if there were episodes of a temporary nature during this period, the duration of symptoms was relatively short compared to the duration of the active period.

One last mention is that the diagnosis of Schizophreniform Disorder is presented as an additional one in case of existing disorders such as the autist one or pervasive of development if and only if delusional ideas and / or hallucinations are present for at least a month.

\section{Schizoaffective disorder}

Historic: Kasanin (1933) introduces the term, to describe patients with symptoms of schizophrenia and affective symptoms, characterized by sudden onset, adolescents, with good premorbid functioning, the presence of a precipitating factor, family medical history of mood disorders.

As a current status, the question arises whether: it belongs to schizophrenia (but patients have prominent affective symptoms); belongs to mood disorders (but there are also prominent symptoms of schizophrenia) (Stefansson, 2009); it is simultaneously an expression of schizophrenia and mood disorders; it is another type of psychosis. More likely it is a heterogeneous group that includes the previous variants (Talbot, 2004).

As a group, patients with schizoaffective disorder have a better prognosis than those with schizophrenia and more reserved than those with mood disorders, and the evolution is rather without deterioration. They respond to timoregulators.

Based on ICD 10, the diagnosis states: "It depends on the approximation of the balance between the importance of schizophrenia (SK) and affective symptoms". Meets the criteria for one of the mood disorders. Symptoms "almost identical to those of schizophrenia" must be present most of the time, for a period of at least two weeks. The above two criteria must be met in the same episode and in the same period, at least part of the episode.

The differential diagnosis is made with: schizophrenia, mood disorders, other psychoses, organic or substance-induced disorders.

Based on DSM-IV diagnosis: an uninterrupted period of illness in which, at some point, a depressive or manic or mixed episode occurs, coexisting with symptoms that meet the A criteria for schizophrenia (the depressive episode must include depressive mood). During the same period of illness there were delusional ideas or hallucinations for at least two weeks, in the absence of prominent emotional symptoms. Symptoms that meet the criteria for Mood Disorder are present for a substantial period of the total duration of the disease (Trifu, 2000).

ICD-10 discusses: Manic-type schizoaffective disorder, mixed-type schizoaffective disorder, depressive-type schizoaffective disorder. DSM-IV-R refers to: Bipolar schizoaffective disorder (manic episode, mixed $+/-$ depressive), Depressive schizoaffective disorder. 


\subsection{Explaining the diagnostic criteria}

Unlike simple schizophrenia, it includes a continuous period in which symptoms typical of an affective disorder exist, along with the symptoms of the first criterion for schizophrenia. During the same period, there were delusions and/or hallucinations for at least two weeks, in the absence of mood swings. These mood symptoms existed for a significant period in the residual or active phase of the pathology. The disorder is not due to substance use or medical conditions generated. For a definite diagnosis it is very important to have evidence related to the patient's past (such as psychiatric reports, previous evaluations).

Criteria for Schizoaffective Disorder manic episode: prominent symptoms of schizophrenia and mania; mood disorders: expansiveness, increased self-esteem, ideas of grandeur or agitation, irritability, aggressive behaviour, ideas of persecution. In both cases hyperactivity, hypoprosexia, and instinctual disinhibition are associated. Symptoms such as: reference ideas, grandeur, persecution can be found. Symptoms "typical" of schizophrenia are needed for diagnosis: thought transmission, ideas of influence, mental automatism, bizarre delusional ideas. They are usually florid psychoses, with sudden onset and complete remission.

Criteria for Schizoaffective Disorder depressive episode: depressive mood associated with other depressive symptoms: psycho-motor retardation, insomnia, decreased appetite, weight loss, decreased interest, hypoprosexia, ideas of guilt, uselessness, ideas of suicide. These are found along with symptoms of schizophrenia: transmission of thoughts, ideas of influence, control, ideas of persecution, pursuit, threatening commentary hallucinations. The episodes are less florid, the duration is longer, the prognosis is more reserved, sometimes the remission is done with a defect (Crow, 2011).

Psychotic disorder due to a somatic disease or related to substance use/abuse; Recurrent depressive disorder - severe depressive episode with psychotic elements; Bipolar disorder (severe depressive or severe manic episode) with psychotic elements; Delusional disorder with depression (Crașovan, 2013).

Evolution and prognosis: between schizophrenia and mood disorders. Manic-type schizoaffective disorder has a similar prognosis to bipolar I disorder. Depressive schizoaffective disorder has a similar prognosis to schizophrenia (Torrey, 1994).

Negative prognostic factors: poor premorbid adaptation, insidious onset, early onset, lack of a precipitating factor, predominance of psychotic symptoms, especially negative ones, hereditary antecedents for schizophrenia. The risk of suicide is $10 \%$, possibly higher in women than in men.

The treatment strategies: hospitalization, drug treatment, psycho-social interventions, drug treatment: antidepressants / thymo-stabilizers + antipsychotics (adapted to symptoms). If thymo-stabilizers are not effective, antipsychotics are required. In practice, antipsychotics are associated with thymo-stabilizers. Bipolar schizoaffective disorder requires: lithium salts, Carbamazepine, Valproate, alone or even in combination. Depressive schizoaffective disorder requires: antidepressants, electroconvulsive therapy. Emphasis on the treatment of emotional symptoms. In practice, antipsychotics are added (Stahl, 2014).

Clinical example: A patient who, at some point in the course of his disorder, has only psychotic symptoms, some disorganization and some aspects of negative phenomenology - noting that it is typical that they are less severe in schizoaffective disorder. The person in question was not manic, dysphoric or hostile, portraying very well the picture of a paranoid schizophrenia, due to the dominant presence of hallucinations and delusional ideas. After a year or two, the same patient arrived in psychiatric care, and for two to three weeks he had no symptoms productive for schizophrenia, such as hallucinations or delusions, disorganized or catatonic behaviour, the only symptom present being that of mood swings, his picture being described as a combination of hostility and emotional lability. The diagnosis of affective schizophrenia involves, for such a patient, over the years, the presence of the third type of episodes, those that simultaneously bring together both disorders of the psychotic register and mood swings. Such a diagnosis can be made with certainty due 
to a good history and the study of older psychiatric medical documents by a doctor who knows the patient's past well.

\section{Delusional disorder}

This disorder is present in two forms: delusional disorder (paranoia) and induced delusional disorder

Generalities: in the past it was called paranoia (from the Greek language). Kraepelin considers paranoia to be a persistent delirium, without hallucinations and damage, systematized paranoid delirium. The predominant symptoms are delusional ideas (systematized, non-bizarre, plausible).

Epidemiology. Etiology. It is a rare disease in which subjects do not seek medical help, the average age of onset is 40 years (18-90), biological factors suggest the involvement of the limbic system and basal ganglia (Waller, 1990).

If we look at ICD-10 diagnosis: an idea / set of delusional ideas, other than those considered specific to schizophrenia (other than the totally impossible ones); such as for example: of persecution, grandeur, hypochondriac, jealousy or erotic. The delirium must last at least three months. There are no persistent hallucinations (or they may occur transiently and be directly related to the delusional topic). The differential diagnosis is made with: schizophrenia, mood disorders (depression), organic or substance-induced. Specifications for subtypes are: persecution, litigation, selfreference, grandeur, hypochondriac (somatic), jealousy, erotomania.

The DSM-IV-TR diagnosis states: non-bizarre delirium (possible: ideas of pursuit, poisoning, infection, being loved, cheated, hypochondriac ideas) lasting at least a month. Criterion A for schizophrenia (hallucinations, delirium, disorganization of thought and activity, negative symptoms) was never met; tactile, olfactory hallucinations related to delirium may occur. Apart from the impact of delirium, social functioning is not affected. Subtypes in DSM-IV-TR: erotomania, grandeur, jealousy, persecution, somatic (hypochondriac), mixed. Other delusions (unspecified delusional disorders): Capgras syndrome; Fregoli syndrome; Cotard syndrome (delusion of negation).

\subsection{Explanation}

Non-bizarre delirium, which refers to realistic, real-life situations lasting at least a month. In this case, the paranoid "force" is much stronger than that felt in schizophrenia.

Criterion A for schizophrenia has never been met, but it is important to note that tactile and olfactory hallucinations, as long as they are related to the delusional theme, may exist (may be real events from which the patient's observation starts, which in a later time are interpreted with delusional valence). Delusional disorder is one that occurs primarily in the plane of thought, not in the perceptual plane (Lewis, 2005).

Apart from delirium, the person's functioning is not markedly affected or impaired, as is the case of behavioural area. An example of unaffected operation is maintaining a high efficiency in several plans (for example, at work). The duration of potential mood episodes parallel to the delirium was shorter than his/her delirium. We exclude the consumption of substances or general medical conditions as a cause of the symptoms.

\subsection{Clinical point of view}

Appearance: correct attire or eccentric, suspicious, hostile patients who may appear normal, except for the delusional system. The clinician should not claim to accept delirium. At the level of perception, patients do not have persistent hallucinations and/or illusions. The DSM-IV-R describes tactile, olfactory hallucinations consistent with delirium. Rare: auditory hallucinations. Attention, memory and orientation are within normal limits. The mood is consistent with delirium (grandeur + euphoria; persecution + suspicion); sometimes depressive symptoms occur. Thinking involves 
content disorders: systematized delirium, possible content (cheated, infected, loved), frequently logical speech; sometimes circumstantiality. The veracity/falsity of the delirium must be checked. The judgment and consciousness of the disease are absent; usually patients are brought to the hospital by the police, family, colleagues. Impulse control: the clinician must assess the risk of suicide, homicide, other types of aggression, caused by delirium. (Trifu, 2000)

As types, we shall mention delusional disorders:

- erotic / erotomania- If the person in question falls in love with someone in his/her immediate entourage or with someone who is a public or important person in social life. The delusional belief that he/she is loved by a well-known, famous person. Erotomania is related to Kandinsky Clerambault mental automatism syndrome. It is a sectoral delirium, which develops "in the network". Clinically, it is more common in women than in men. Forensic: men risk homicide vis-à-vis the person at the centre of the delirium. Those involved are often isolated, alone, with a poor sex life. Classically the phases of: hope, disappointment, resentment are described. Efforts are made to contact the person who represents the object of the delirium (phone, letters, gifts, visits, supervision, follow-up), the person in question interpreting any gesture (rejection) as a proof of love. The risk of hetero-aggressive acts should not be omitted.

- of grandeur- The person acquires an important but unlikely status, the delirium being fixed on fantastic, heroic characteristics, histrionic elements, but with delusional value. We're talking about megalomania. Examples: the person in question considers that he/she has a special but unrecognized talent or that he/she has discovered an invention that he/she is trying to popularize or that he/she has a relationship with a high-ranking person. When the content of the delirium is a religious one, the person in question may enroll to various sects.

- of jealousy- Delirium implies an absolute conviction that the loved one is cheating on him /her - attitudinal we find: checking messages, different tracking behaviours. It is also called conjugal paranoia, Othello syndrome (when referring to husband/wife). It is more common in men. The onset may be sudden; the disorder may subside if those involved break up. There is a risk of hetero-aggression, abuse, limitation of autonomy. It can be associated with alcohol addiction (von Krafft-Ebing). Subjects gather "evidence" to support delirium (they make investigation, follow the person).

- persecution - dangerous clinical situation from two perspectives. One is when the person in question has a moment in which he/she transforms from persecuted to persecutor, the consequence being represented by possible acts of heteroaggressiveness, which can go as far as killing, from the delusional conviction that it is better for him/her to attack his/her persecutors than to be him/her the one persecuted. The moment of this inner transformation is doubled by an attitude of emphasis, superiority and the feeling that he/she no longer of use to expose his/her anxieties or paranoid theories, because he/she is not understood anyway (Minca, 2013). The second risk is that of suicide attempt (although the literature cites them as rarer in delusional disorder), if the patient is convinced that he/she is almost constantly pursued by persecutors who want to kill him/her by subjecting him/her to multiple tortures and, due to the depression following the delusional idea of pursuit, persecution and harm (which is one of micromaniac influence), the patient will take the decision to commit suicide because he/she cannot "get rid" of his/her persecutors; and then he/she decides to kill himself in a quick way, rather than let himself be killed in torment. (Kaplan, 2009)

One or more related topics are of: conspiracy, deception, prosecution, espionage, poisoning, harassment, hindered in pursuing certain goals. Small problems can be exacerbated (and taken as "evidence"), which later become the focus of a delusional topic. Sometimes legal redress is attempted: querulent paranoia, the one in question resorting to numerous lawsuits. There is a risk of verbal or physical aggression. The paranoid no longer has the ability to doubt, he/she builds through interpretations a delusional scenario with a persecutory topic. The delusional topics most at hand are: the professional environment, the neighbors. After a phase of elaboration (months, years), in which the person in question accumulates material for future interpretations, the revelation, the absolute certainty appears; hence the delirium develops in the 
network. With age, a resignation may occur: "The paranoid does not heal, he disarms".

- somatic- If the hypochondriac manifestations acquire a delusional value. It is also called hypochondriac monosymptomatic psychosis. The differential diagnosis is made with hypochondria, a situation in which the degree of conviction of the patient matters. Examples: infection, insect infestation on/in the skin; dysmorphophobia; body odours (skin, mouth, vagina); certain parts of the body in relation to which the patient is convinced that they no longer function. There is also a risk of suicide. Sometimes we find a history of substance abuse or CCT.

- mysticism - In which delirium involves communication with various entities or at various levels (eg spiritual).

- we shall mention a delusional form focused on self-destruction - Delirium implies the urgent need of the person to injure himself in violent, brutal, more or less deadly ways.

Psycho-social theories in Delusional Disorder support the existence in personal life history of factors such as: physical and mental abuse, cruel, messy parents, who cannot be relied on, demanding, perfectionist climate, hostile, dangerous environment (E. Erikson), which leads to the lack of basic trust, hearing loss, social isolation, loneliness, immigration.

Projective psychological tests highlights: concerns with paranoid, grandiose topics, inferiority, inadequacy, anxiety (Badner, 2002)

Psychodynamic factors bring into question the following defences used: denial (denying shame, humiliation, inferiority), reaction formation (transformation of unacceptable feelings into their opposite, such as inferiority is transformed into grandeur), projection (major defence, through which unacceptable feelings are projected on others). (Sullivan, 2003)

\subsection{Psychodynamic factors}

Such people are hypersensitive, with specific defence mechanisms: reactionary formation, projection, non-denial. Freud stated that those concerned defend themselves from unconscious homosexual tendencies by non-denial and projection. "I love him" becomes "I do not love him, I hate him," after denying having the projection: "I do not hate him, he hates me," which leads to "I am persecuted by him." Erotomania delirium: male patients "I love him" turns into "I love her" and leads to "she loves me." The delusion of grandeur: "I don't love him" becomes "I love myself." The jealousy delusion: "I don't love him" becomes "she, his wife, loves him".

These hypotheses are not a sufficient explanation. Cameron discusses situations that favour the development of delusional disorder (they find the explanation in the delusional system): those concerned expect sadistic treatment, are dominated by distrust and marked suspicion, social isolation, situations that accentuate envy, jealousy or situations that decrease self-esteem or that favour rumination about explanations, motivations. (Lieberman, 2005) Distrust is possibly related to a constantly hostile family environment. Defence mechanisms are: reactionary formation (against aggression, the need for addiction, affection), denial (avoiding awareness of the painful reality), projection (when the subject defends him/herself from his/her own aggressive, unacceptable impulses). Hypersensitivity, feelings of inferiority and defence mechanisms lead to delusion of grandeur. (Perkins, 2005)

\subsection{Therapeutic perspective}

As a therapist, I listen to the person in question when they express their concern and respond with understanding to the fact that delirium is disruptive and intrusive. I help the patient find a way to live more comfortably with his delirium. I understand that this is a means of dealing with shame and inadequacy; I understand his/her sensitivity to any insult. (Taylor, 2009) Psychosis is a defensive response for someone with inadequate coping mechanisms, and psychotherapy should focus on the significance of life events and the formation of more adaptive, less devastating coping 
mechanisms. Discretion and honesty are important, because these patients are hypervigilant of deception and insincerity. (Hodgkinson, 2004) The stresses that triggered the first delirium matter, and the case should always be considered from the perspective that other stresses can exacerbate delusional symptoms. The patient must be helped to develop the means to cope with stress, while the therapist must find out where the shame and inadequacy comes from. (el-Husseini, 2002)

Clinical example 1: A 74-year-old patient with Axis II comorbid diagnosis Organic personality disorder (and at the same time classified as disabled), presents a decompensation of type Delusional disorder persecutor type on organic background (therapeutically uncontrolled diabetes) where her reference ideas were related to the loss of her pension and to the fact that her granddaughter was persecuting her.

Clinical example 2: A patient of about 40 years has disorders of sexual dynamics (impotence type), possibly potentiated by the side effects of treatment for a bipolar affective disorder, maintenance doses. Against this background, the person in question developed a jealousy delusion towards his wife's boss, being convinced that these two are living together in an extramarital affair. The wife, a beautiful woman, had several moments when she could not help herself and burst into tears during the consultation, assuring the psychiatrist that this was just a delusional conviction of her husband. The couple also had a child. In accordance with this belief, the behaviour of the person in question also changes, as he installed a software on his wife's phone, which allowed him to follow the messages sent and received by her, as well as to record the conversations. The patient said that in one of the recordings he heard his wife complaining about the marriage and then an exchange of amorous remarks between her and the boss followed. Also, in the same conversation, apparently, her boss suggested that she put "something" in the patient's coffee, so that she would not be beaten by her husband, if she decides to call an ambulance and law enforcement bodies.

Clinical example 3: A 46-year-old patient (treated both medically and therapeutically) is convinced that her husband is cheating on her and that he wants to live a double life. She did not want to give up the relationship because of her status (lawyer) and apparently because of her pride and children. This belief led her to spend a lot of money on tracking methods, private detectives, devices by means of which to check her husband's phone, and the fact that she spent hours alone in the car in front of various locations to wait and interpret the presence of the husband. in that place. Apparently, the fact that her husband cheated on her was confirmed countless times, but the woman in question did not stop consuming money and time to follow him, this also having consequences for the children. In this case, even if her initial suspicions are confirmed, delusional disorder begins, according to some psychiatrists, from the moment she can no longer stop the pursuit behaviours.

The differential diagnoses: paranoid schizophrenia: delirium (+/- bizarre), hallucinations, disorganized way of thinking, disorganization of activity, negative symptoms, marked impairment of functioning; delusional disorder: non-bizarre delirium, without hallucinations or they are rare, without formal thinking disorders, with relatively good functioning except for the area of delirium; mood disorders: depression /mania with psychotic elements; Schizoaffective disorder; organic disorders; substance-induced disorders; paranoid personality disorder (frequently premorbid - 50\%; pride, high self-esteem, distrust, false judgments; psychorigidity; social maladaptation)

\subsection{Evolution and prognosis}

Sometimes there are precipitating factors: recent emigration, social conflicts, social isolation. Frequently the onset of delirium is apparently sudden. The intelligence of the person in question is below the average of intelligence, the premorbid personality is hypersensitive, dominant. The symptoms may fade in weeks, months, years, but may be triggered again, possibly due to a precipitating factor. There is a risk of depression and suicide risk: depressive reactions are relatively common, facilitated by a relative awareness. Depressive symptoms of chronic delirium are a psychiatric 
emergency. The encystation of delirium takes place in the case of favourable evolution, delusional beliefs are isolated, without being criticized.

Good prognostic factors: good occupational, social, integration, female onset under 30 years, sudden onset, short duration of the episode, the presence of precipitating factors. Those with persecution, somatic, erotic delirium would have a better prognosis than those with delirium of grandeur and jealousy.

\subsection{Therapeutic principles}

Initially: establishing an alliance with the patient. The doctor must remain aware of the risk of being included in the delusional system of the patient. The long-term therapeutic approach depends on: the evolution of the delusional disorder under treatment, the family and socio-professional context, the degree of danger, the risk of disinsertion of the patient.

Pharmacotherapy - treatment of choice: antipsychotics. Usually, the patient refuses the treatment, integrates it into the delusional system. The doctor should try to explain to the patient the need for treatment, the role of the law (commission).

Psychotherapy: essential - establishing a therapeutic, trusting relationship with the patient; establishing and respecting the framework. Individual therapy must be oriented on insight, of a cognitive, behavioural nature. Initially, the therapist does not contradict, but does not accept delirium. It should be noted that the patient's internal experience is that of the victim of a world that persecutes him/her. The possibility of family therapy should also be evaluated.

Induced psychotic disorder

The cause is primarily psychogenic. Psychological factors postulate an isolated relationship from a social point of view: the first partner is dominant, with the delusional system formed, and the other is submissive and dependent. Psychodynamic: the dominant one keeps in touch with reality through the submissive one, the submissive one seeks the care and acceptance of the dominant one, and the two have a strong ambivalent relationship.

\section{Conclusion}

It should be mentioned that in brief psychoses, the factors of psychogenic reactivity are significant, and the prognosis can be good, especially since the onset is more flourishing, it is consumed faster, there are favourable circumstances for its occurrence, premorbid personality is a quality one, and insight appears early with the onset of chemical restraint. Vulnerability in psychosis is part of the "puzzle". Referring to the thinking and emotionality of the schizophrenic or, in some cases, of the delusional patient, there are in the conception of various authors symptoms of rank I or rank II, depending on which the prognosis is milder or more severely affected.

\section{References}

1. American Psychiatric Association. (2013). Schizophrenia Spectrum and Other Psychotic Disorders in Diagnostic and statistical manual of mental disorders (5th ed). Washington,

2. Badner, J. A., Gershon, E. S. (2002). Meta-analysis of whole-genome linkage scans of bipolar disorder and schizophrenia. Mol Psychiatry 7, 405-411.

3. Craşovan, D. I. (2013). Psychological defense mechanisms and coping mechanisms in nonpsychotic major depressive disorder. Journal of Educational Sciences \& Psychology, 3(2), 8998.

4. Crow, T. J. (2011). The missing genes: what happened to the heritability of psychiatric disorders? Mol Psychiatry 16, 362-364.

5. Drăgoi, A. M., Rădulescu, I., Năsui, B. A., Pop, A. L., Varlas, V. N., Trifu, S. (2020). Clozapine: An Updated Overview of Pharmacogenetic Biomarkers, Risks, and Safety Particularities in the Context of COVID-19. Brain Sci. 2020, 10, 840. 
6. Grigoriu, M. E., Costea, R. V., Grigoriu, C.I., Furtunescu, F. L. (2018). Endoscopic management of choledocolithiasis related to periampullary duodenal diverticula. Medicalsurgical journal, 122(1), 102-108.

7. Hodgkinson, C. A., Goldman, D., Jaeger, J., Persaud, S., Kane, J. M., Lipsky, R. H., Malhotra, A. K. (2004). Disrupted in schizophrenia 1 (DISC1): association with schizophrenia, schizoaffective disorder, and bipolar disorder. Am J Hum Genet 75(5):862-72.

8. el-Husseini, A. D., Bredt, D. S. (2002). Protein palmitoylation: a regulator of neuronal development and function. Nat Rev Neurosci 3: 791-802.

9. International Statistical Classification of Diseases and Related Health Problems 10th Edition (ICD-10). (2010). Chapter V, Mental and Behavioural Disorders (F00 - F99).

10. Kaplan \& Sadock's. (2009). Comprehensive Textbook of Psychiatry, Ninth Edition, 2 vol, Wolters Kluwer/ Lippincott Williams \& Williams.

11. Lefter, S. (2020). A study on the structure of Self in personality disorders and attachment disorders: narcissistic personality disorder and histrionic personality disorder. Journal of Educational Sciences and Psychology 10(1): 149-157.

12. Lewis, C. M., Levinson, D. F., Wise, L. H., DeLisi, L. E., Straub, R. E., Hovatta, I., Williams N. M., Schwab, S. G., Pulver, A. E., Faraone, S. V. et al. (2003). Genome scan meta-analysis of schizophrenia and bipolar disorder, part II: Schizophrenia. Am J Hum Genet 73(1): 34-48.

13. Lewis, D. A., Hashimoto, T., Volk, D. W. (2005). Cortical inhibitory neurons and schizophrenia. Nat Rev Neurosci 6: 312-324.

14. Lieberman, J. A., Perkins, D., Belger, A., Chakos, M., Jarskog, F., Boteva, K., Gilmore, J. (2001). The early stages of schizophrenia: speculations on pathogenesis, pathophysiology, and therapeutic approaches. Biol Psychiatry 50(11): 884 - 897.

15. Luo, X., Huang, L., Han, L., Luo, Z., Hu, F., Tieu, R., Gan, L. (2014). Systematic prioritization and integrative analysis of copy number variations in schizophrenia reveal key schizophrenia susceptibility genes. Schizophr Bull 40(6): 1285-99.

16. McClellan, J. M., Susser, E., King, M. C. (2007). Schizophrenia: a common disease caused by multiple rare alleles. Br J Psychiatry 190: 194-199.

17. Minca, D. G., Furtunescu, F. L., Calinoiu, G., Domnariu, C. D., Costea, R. V. (2013). Profile of persons involved in traffic accidents in Romania. Romanian journal of legal medicine 21(2), 155-160.

18. Neagu, S., Lerescu, L., Costea, R., Tucureanu, C., Caras, I., Gangura, G., Pitica, R., Salageanu, A. (2012). Perioperative immunological changes in colorectal cancer patients. Chirurgia 107(1), 59-65.

19. Perkins, D. O., Gu, H., Boteva, K., Lieberman, J. A. (2005). Relationship between duration of untreated psychosis and outcome in first episode schizophrenia: a critical review and metaanalysis. Am J Psychiatry 162: 1785-1804.

20. Popa, C. C., Badiu, D. C., Andronache, L. F., Costea, R. V., Neagu, S. I., Stoian, A. P., Socea, B., Ionescu, D. (2019). Differential Diagnosis in Esophageal Cancer Review on literature. Rev. Chim. 70(1), 331-335.

21. Pulver, A. E., Liang, K., Brown, C. H., Wolyniec, P., McGrath, J., Adler, L., Tam, D., Carpenter, W. T. Jr., Childs, B. (1992). Risk factors in schizophrenia: Season of birth, gender and familial risk. British Journal of Psychiatry 160: 65-71.

22. Rujescu, D. (2017). Suche nach Risikogenen bei Schizophrenie. Nervenarzt 88: 751-754.

23. Saha, S., Chant, D., Welham, J., McGrath, J. (2005). A systematic review of the prevalence of schizophrenia. PLoS Med 2: e141.

24. Sîrbu, R. C., Glăvan, D. G., Duică, L. C., Strunoiu, L. M., Sîrbu, A. A. M., Bădescu, Ș., Cara, M. L., Pîrlog, M. C. (2020). The role of psychotrauma in the onset and the evolution of the paranoid schizophrenia. Journal of Educational Sciences \& Psychology 10(2): 112-124.

25. Stahl, S. M. (2013).The Prescriber's Guide.

26. Stahl, S. M., Stahl's (2014). Essential Psychopharmacology, Fourth Edition.

27. Stefansson, H, Ophoff, R. A., Steinberg, S., Andreassen, O. A., Cichon, S., Rujescu, D., Werge, T., Pietiläinen, O. P., Mors, O., Mortensen, P. B. et al (2009). Common variants conferring risk of schizophrenia. Nature 460(7256): 744-7.

28. Sullivan, P. F., Kendler, K. S., Neale, M. C. (2003). Schizophrenia as a complex trait: evidence from a meta-analysis of twin studies. Arch Gen Psychiatry 60: 1187-1192.

29. Talbot, K., Eidem, W. L., Tinsley, C. L., Benson, M. A., Thompson, E. W., Smith, R. J., Hahn, C. G., Siegel, S. J., Trojanowski, J. Q., Gur, R. E. et al. (2004). Dysbindin-1 is reduced in intrinsic, glutamatergic terminals of the hippocampal formation in schizophrenia. J Clin Invest 113(9): 1353-63. 
30. Taylor, D., Paton, C., Kapur, S. (2009). The Maudsley, Prescribing Guidelines, 10th Edition, The South London And Maudsley NHS Foundation Trust Oxleas NHS Foundation Trust 2009, Informa Health Care.

31. Tereanu, C., Minca, D. G., Costea, R., Janta, D., Grego, S., Ravera, L., Pezzano, D,, Vigano, P. (2011). ExpIR-RO: A Collaborative International Project for Experimenting Voluntary Incident Reporting in the Public Healthcare Sector in Romania. Iranian journal of public health 40(1), 22-31.

32. Torrey, E. F., Bowler, A. E., Taylor, E. H., Gottesman II. (1994). Schizophrenia and Manic Depression Disorder: The Biological Roots of Mental Illness as Revealed by the Landmark Study of Identical Twins. Basic Books, New York.

33. Trifu, S., Marica, S., Brăileanu, D., Carp, E. G., Gutt, A. M. (2015). Teaching Psychiatric Concepts of Neurosis, Psychosis and Borderline Pathology. Conceptual Boundaries. Procedia Social and Behavioral Sciences 203: 125-129.

34. Trifu, S., Trifu, A. D. (2020). Receptor profiles of atypical antipsychotic molecules. U.P.B. Sci Bull 82: 113-128.

35. Trifu, S. C., Vlăduţi, A., Trifu, A.I. (2020). Genetic aspects in schizophrenia. Receptoral theories. Metabolic theories. Rom J Morphol Embryol, 61(1):25-32.

36. Trifu, S., Tudor, A., Radulescu, I. (2020). Aggressive behavior in psychiatric patients in relation to hormonal imbalance (Review). Experimental and Therapeutic Medicine, 20(4), 3483-3487.

37. Waller, N. G, Kojetin, B. A., Bouchard, T. J. Jr, Lykken, D. T., Tellegen, A. (1990). Genetic and environmental influences on religious interests, attitudes, and values: A study of twins reared apart and together. Psychological Science 1: 138-142.

38. Weickert, C. S., Straub, R. E., McClintock, B. W., Matsumoto, M., Hashimoto, R., Hyde, T. M., Herman, M. M., Weinberger, D. R., Kleinman, J. E. (2004). Human dysbindin (DTNBP1) gene expression in normal brain and in schizophrenic prefrontal cortex and midbrain. Arch Gen Psychiatry 61(6): 544-55.

39. Williams, H. J., Owen, M. J., O'Donovan, M. C. (2007). Is COMT a susceptibility gene for schizophrenia? Schizophr Bull 33(3): 635-41.

40. Zai, C. C., Manchia, M., Zai, G. C., Woo, J., Tiwari, A. K., de Luca, V., Kennedy, J. L. (2018). Association study of BDNF and DRD3 genes with alcohol use disorder in Schizophrenia. Neuroscience Letters, 671, 1-6. 